\title{
Importance of the single-particle continuum in BCS pairing with a pseudostate basis
}

\author{
J. A. Lay ${ }^{1}$, C. E. Alonso ${ }^{2}$, L. Fortunato ${ }^{1}$, and A. Vitturi ${ }^{1}$
}

${ }^{1}$ Dipartimento di Fisica e Astronomia "Galileo Galilei", Università di Padova, and INFN, Sezione di Padova, via Marzolo, 8, I-35131 Padova, Italy

${ }^{2}$ Departamento de Física Atómica, Molecular y Nuclear, Facultad de Física, Universidad de Sevilla, Apartado 1065, 41080 Sevilla, Spain

\begin{abstract}
In a recent work [arXiv:1510.03185] the use of the Transformed Harmonic Oscillator (THO) basis for the discretization of the singleparticle continuum into a Generalized Bardeen-Cooper-Schrieffer (BCS) formalism was proposed for the description of weakly bound nuclei. We make use of the flexibility of this formalism to study the evolution of the pairing when the nucleus becomes more and more weakly bound. Specifically we focus on the evolution of the occupation of the different partial waves in ${ }^{22} \mathrm{O}$ when the Fermi level approaches zero.
\end{abstract}

Present developments in nuclear facilities allow to study new exotic nuclei, approaching the proton and neutron driplines for both light and medium mass regions of the Segrè Chart. Nuclei close to the dripline are weakly bound and, therefore, affected by the continuum, situations where the last nucleon or nucleons are no longer bound and can explore distances far from the rest of the nucleus.

The effects of the continuum have been traditionally explored for light nuclei where the proximity of the driplines provides a rich variety of weakly 
bound nuclei and experimental data. In the medium mass region, several attempts for generalizing mean field approaches have been proposed with different results [1,2]. Regarding the Bardeen-Cooper-Schrieffer (BCS) approximation, the single-particle continuum is first discretized and then treated normally splitting resonant and background contributions or including a continuum single-particle level density [2].

In a recent work [3] we studied the possibility of treating background and resonant parts of the continuum on the same footing. In order to do so, the single-particle continuum is discretized using a square-integrable basis. The pseudostates or eigenstates obtained through the diagonalization of the matrix elements of the single-particle Hamiltonian are expected to give a good representation of the continuum. Then, the pairing Hamiltonian is written in terms of the pair creator and annihilator operators built on top of these pseudostates. The strength of the pairing is determined by a densitydependent delta interaction and matrix elements are calculated separately for each couple of pairs. Once we set a maximum energy, it is possible to solve the BCS equations.

The basis chosen for this purpose is the Transformed Harmonic Oscillator (THO) basis. It is constructed by applying a Locale Scale Tranformation to the Harmonic Oscillator basis of the form [4]:

$$
s(r)=\left[\frac{1}{\left(\frac{1}{r}\right)^{m}+\left(\frac{1}{\gamma \sqrt{r}}\right)^{m}}\right]^{\frac{1}{m}},
$$

that depends on the parameters $m$ and $\gamma$. Even though we increase the number of parameters with respect to the simpler $\mathrm{HO}$, two main advantages are obtained: the asymptotic behaviour of the functions is now exponential, rather than gaussian as in the $\mathrm{HO}$ case, and $\gamma$ can be used to tailor the basis to the different cases. This is of great help when assessing the importance of the low-energy continuum since, by reducing the value of $\gamma$, the pseudostates tend to concentrate at low energies, thus optimizing the calculation.

Already in [3], an interesting increase of the occupation of the nonresonant low-energy continuum was found for extremely weakly bound situations. In this work, we will concentrate on the evolution of the total occupation when we vary the strength of the single-particle continuum. Remember that, in the BCS theory, the quantities $v^{2}$ and $u^{2}$ stand for the occupation and deoccupation of the different level. We will choose the same case and parameters chosen in [3], so that we will look to the occupations in ${ }^{22} \mathrm{O}$ when varying the strength of the central single-particle potential $V$. 


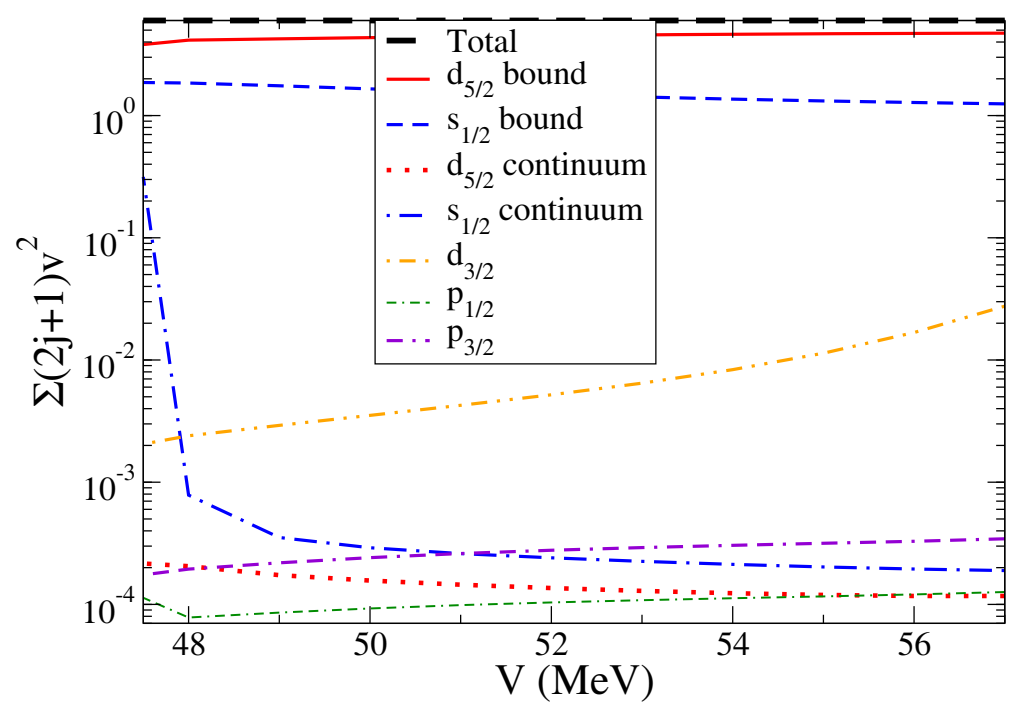

Figure 1: Occupation corresponding to the six valence neutrons considered in ${ }^{22} \mathrm{O}$ for the different partial waves. We separate the $d_{5 / 2}$ (solid line) and $s_{1 / 2}$ (dashed line) bound contribution from the corresponding $d_{5 / 2}$ (dotted line) and $s_{1 / 2}$ (dotted dashed line) continuum contribution. The thick dashed line corresponds to the total occupation as the sum of the different contributions considered which, by construction, is always the number of valence neutrons.

In [3], the pairing interaction between the different pairs is calculated by using a density dependent delta interaction (DDDI) fitted to reproduced the neutron separation energy in ${ }^{18} \mathrm{O}$, and then renormalized along the isotopic chain following a $1 / A$ reduction.

In fig. 1 we show the occupations in ${ }^{22} \mathrm{O}$ for all the partial waves considered, calculated for different depths of the central single-particle potential $V$. We have also considered separately the bound and continuum parts of the occupation in the $d_{5 / 2}-$ and $s_{1 / 2}$-waves. It is commonly stated that the contribution of the continuum is mainly governed by the resonances present in each case. In our case, this fact should be reflected in the dominance of the $d_{3 / 2}$ contribution where a low-lying resonance can be found. This is true for a well-bound ${ }^{22} \mathrm{O}$, which in fig. 1 corresponds to the right part of the figure around $V=57 \mathrm{MeV}$. It is interesting to see how the occupation decreases when the strength of the potential is reduced. However, the occupation of the resonant part of the continuum is still larger by more than one order of magnitude than the non-resonant part of the continuum.

This situation changes for really weakly-bound situations. A sudden increase of the $s_{1 / 2}$ continuum contribution is found, even though only for 
an extreme situation. One has to keep in mind than the Fermi level for $V=47.5 \mathrm{MeV}$ is practically zero. On the other hand, the bound part of the $s_{1 / 2}$ contribution is more or less constant with a slight increase.

If we look at the remaining contributions, only the $d_{5 / 2}$ continuum part is also constantly increasing when reducing the strength $V$. However, this represents a modest variation if we compare with the behaviour of the $s_{1 / 2}$ waves. Nevertheless, one has to remind that we are using a DDDI, which can overestimate the relative importance of the $s$-wave in detritment of contributions with larger orbital angular momentum. A more realistic treatment of the pairing interaction could lead to a larger increase of the occupation of the $d_{5 / 2}$ continuum, at least in comparison with the $s_{1 / 2}$ contribution.

The fact that only those continuum components with a bound state of the same orbital and total angular momentum show such an increase is telling us that this effect is not related with the extension of the wave functions as in the case of the formation of a halo nucleus. In such a case, $p_{3 / 2}$ occupation should show an increase since the extension of $p$-waves also goes to infinity when the binding energy goes to zero. Instead, they decrease as we can see in fig.1.

In conclusion, non-resonant continuum contributions to the ground state of a nucleus cannot be neglected when dealing with weakly bound nuclei. The importance of the resonant configuration decreases consistently when the system becomes more and more weakly bound until a situation is reached where is no longer the dominant part of the single-particle continuum. However, we also show that for not so extreme situations, it is the resonant part that carries almost all the importance of the continuum.

\section{Acknowledgments}

This work has been supported by MIUR research fund PRIN 2009TWL3MX, by the Spanish Ministerio de Economía y Competitividad under Project FIS2014-53448-C2-1-P by Junta de Andalucía under group number FQM160 and Project P11-FQM-7632, and by the Consolider-Ingenio 2010 Program CPAN (CSD2007-00042). The research leading to these results has received funding from the European Commission, Seventh Framework Programme (FP7/2007-2013) under Grant Agreement $\mathrm{n}^{\circ}$ 600376. J.A.L. is a Marie Curie Piscopia fellow at the University of Padova. 


\section{References}

[1] J. Dobaczewski et al., Phys. Rev. C, 53 (1996) 2809.

[2] R. Id Betan, Nucl. Phys. A, 879 (2012) 14.

[3] J. A. Lay et al., arXiv:1510.03185 Preprint, 2015.

[4] J. A. Lay et al., Phys. Rev. C, 82 (2010) 024605. 